\title{
Direct Power Control of A Multilevel Inverter Based Active Power Filter
}

\author{
George Zhou \\ Honeywell , Engines \& Systems \\ 3333 Unity Drive \\ Mississauga, Ontario \\ Canada L5L 3S6 \\ E-mail: george.zhou2@honeywell.com
}

\author{
Bin $\mathrm{Wu}$ \\ Ryerson Univ. Dept. of E\&CE \\ 350 Victoria Street \\ Toronto, Ontario \\ Canada M5B 2K3 \\ E-mail: bwu@ee.ryerson.ca
}

\author{
Donglai Xu \\ School of Science \& Technology \\ Univ. of Teesside \\ Middlesbrough, Tees Valley \\ TS1 3BA UK \\ Email: d.xu@tees.ac.uk
}

\begin{abstract}
This paper proposed a cascaded H-bridge multilevel inverter based direct connected active power filter for medium/high voltage application without using the bulky transformer or passive filter. Due to the limited switching frequency (typically below $1 \mathrm{kHz}$ ) of high-power solid-state devices(GTO/IGCT), multiple sychronous/stationary reference frame current controller are reviewed and derived. To improve the dynamic performance, a hybrid frame struture based curent controller is presented. Furthermore, a direct power controller is then proposed based on the understanding between the Instantaeous Reactive Power (IRP) and Synchronous Reference Frame (SRF) theory. It has clear physical meaning and can be considered as a simplfied version of the hybrid frame current controller. Simulations on a $4160 \mathrm{~V} / 1.2 \mathrm{MVA}$ system and experimental results on a $208 \mathrm{~V} / 6 \mathrm{KVA}$ labrotary prototype are preseted to validate the proposed active power filter design.
\end{abstract}

Keywords: Active power filter, Medium voltage, Direct power control, Current control, Low switching frequency.

\section{Introduction}

Voltage source inverter based parallel active filter is known for current harmonic compensation of the power system and have been widely studied by using high switching power device(IGBT). For medium/high voltage application, a direct connected active filter can be more attractive by eliminating the bulky transformer or passive filter. Due to the limited switching frequency (typically below $1 \mathrm{kHz}$ ) of high-power solid-state devices (GTO/ IGCT), multilevel inverters have to be used[1][2]. This paper proposed a cascaded H-bridge multilevel inverter based active filter. Multiple techniques are studied to increase the system bandwidth at low switching frequency:First, a software based multiple-sampled phase shifted PWM is proposed.

- Secondly, among most current controller designed for active filters, multiple synchronous reference frame based current controller is proven to have the highest bandwidth with modest feedforward gain.

- Thirdly, an equivalent stationary frame based current controller is easily derived based on complex vector theory. The results are similar as that derived from convolution[3] or physical concept[4] in recent literature.

- Fourthly, to remove the fundamental cross-decoupling term in synchronous reference frame, a synchronous / stationary hybrid frame based current controller is proposed.

- Finally, Instantaneous reactive power(IRP) theory and Synchronous reference frame(SRF) based method are briefly compared. The connection between them is explained. Based on this, an instantaneous reactive power theory based direct power control is derived from the synchronous / stationary hybrid frame based current controller.

This new concept combines the IRP theory and current control into one controller called direct power controller. The power reference is created by the IRP and a linear direct power controller is designed accordingly. It is similar to the multiple reference frame current controller, which is suitable for high power/low switching frequency applications. Simulations on a 4160V/1.2MVA system are provided and the proposed theory is verified on a 208V/6KVA five-level cascaded H-bridge inverter based active power filter.

\section{Cascaded H-bridge multilevel inverter based active power filter}

The proposed multilevel inverter based active filters are shown in Fig.1. The design is based on the medium voltage applications $(2.3 \sim 13.8 \mathrm{KV})$.

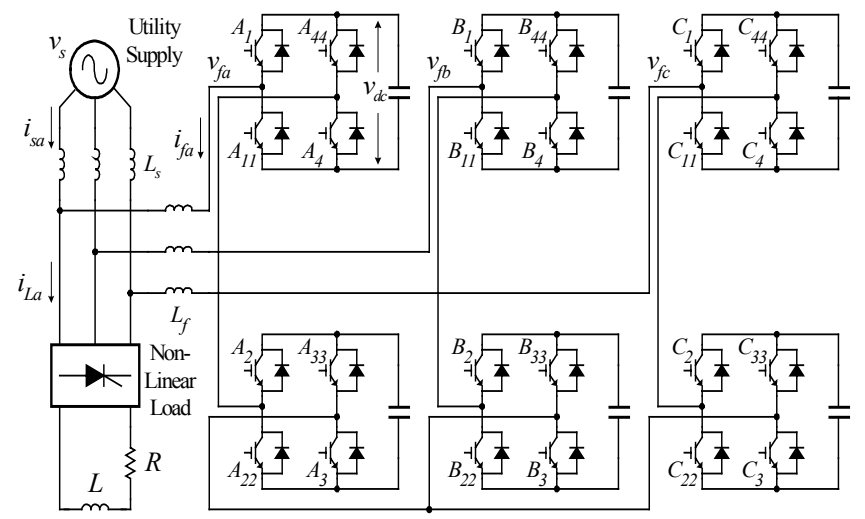

$\mathrm{V}_{\mathrm{s}}($ Line-Line $)=4160 \mathrm{~V}, R=26 \Omega, L=0.15 \mathrm{H}, \mathrm{L}_{\mathrm{s}}=0.002 \mathrm{H} \sim 0.004 \mathrm{H}(5 \% \sim$ $10 \%$ per unit), $\mathrm{L}_{\mathrm{f}}=0.002 \mathrm{H}\left(5 \%\right.$ per Unit), $C=1000 \mu \mathrm{F}, \quad V_{d c} *=1875 \mathrm{~V}$

Fig. 1 The topology of proposed medium voltage multilevel inverter based active power filter

Compared with diode-clamped or flying capacitor multilevel topology, this structure is chosen because it needs the least components[1]. As shown in Fig.1, the converter is composed of six $\mathrm{H}$-bridges and six DC capacitors. It is connected to the power line through a $5 \% \sim 10 \%$ per unit inductor only. 


\subsection{PWM Method}

As shown in Fig.2(a) and Fig.2(b), a software based multiple-sampled phase shifted carried based PWM[2] is used for PWM control. This PWM method allows using high sampling control rates at low switching frequency and is most suitable for the proposed active filter.
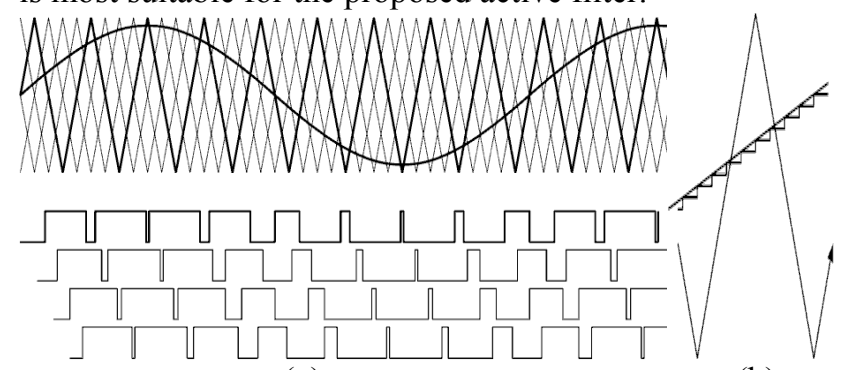

(a)

(b)

Fig.2 Multi-sampled phase shifted carrier based PWM for multilevel inverter

\subsection{Current control}

As shown in Fig.3, current controllers of active power filters have been extensively studied[5][6], but most methods are not suitable for low switching frequency applications. A multiple synchronous reference frame based current control method[7] can be designed to reach zero steady state error for multiple harmonics, therefore it has superior benefits in low switching frequency application where feed-forward gain has to be modest to limit the switching frequency at the carrier frequency. To simplify the complex rotation frame transformation, an equivalent stationary frame current controller can be developed by convolution derivation[3] or the direct physical concept[4]. However, the well-known crosscoupling term in rotation frame is not considered and the relationship between rotation frame and stationary frame is not straightforward to understand.
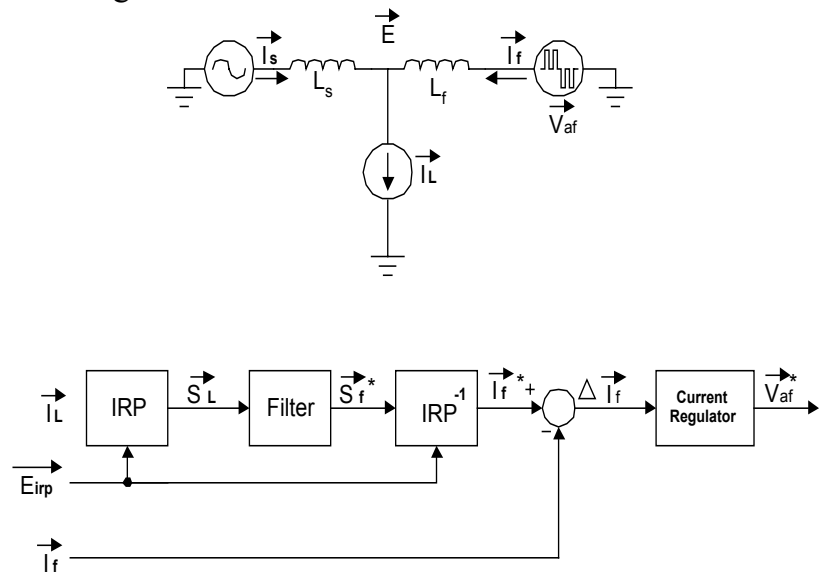

Fig.3 Typical current controlled voltage source inverter based parallel active power filter

A complex vector based mathematical model(Fig.4a) is used to represent the multiple synchronous reference frame based current controller, then a simple and straightforward derivation based on complex vector is used to find the equivalent stationary frame based current controller (Appendix I and Fig.4b). Eq.(5) shows the same result as the ones in [3] and [4] and Eq.(2) gives the general relationship between rotation frame and stationary frame. To consider the cross coupling term at fundamental frequency, a synchronous/stationary hybrid structure is proposed with de-coupling control (Fig.4c and Fig.4d). In this synchronous/stationary hybrid structure, current reference is generated by the well-known instantaneous reactive power(IRP) theory, and then transformed into synchronous reference frame (SRF) for the current control. This two step transformations make the system complicated and a merging of these two transformations can be found. This is based on a brief review of IRP and SRF based system models in the next section.
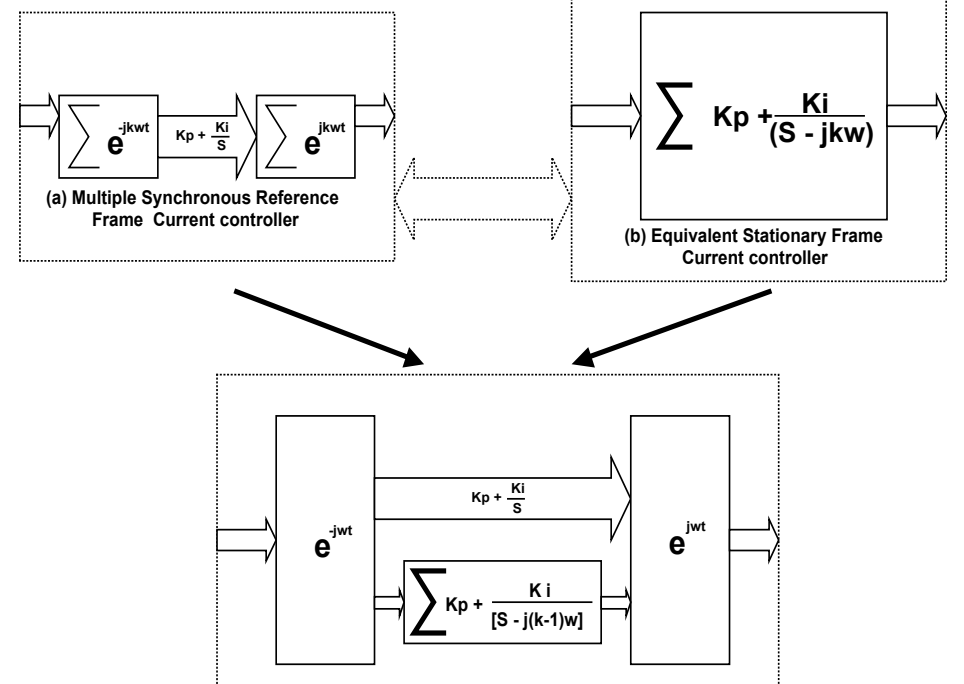

(c) Equivalent Synchronous /Stationary Hybrid Current controller

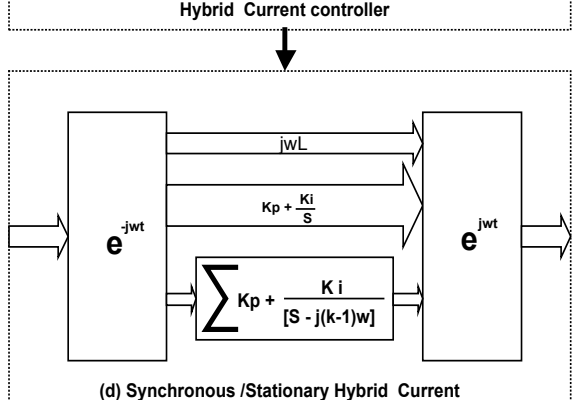

(d) Synchronous / Stationary Hybrid Current controller with fundamental decoupling

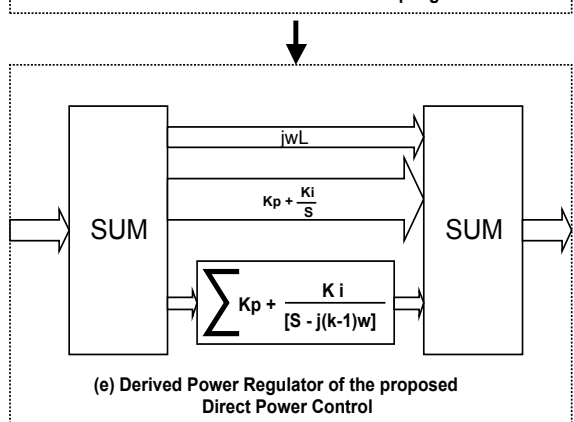

Fig.4 The derivation of direct power control concept

\subsection{System model based on SRF and IRP}

Appendix II and III provide the mathematical model of the active filter based on SRF and IRP theory. The similarities between appendix II and III show that IRP is equivalent as $\mathrm{SRF}$ when $\mathrm{E}_{\text {irp }}$ only has one frequency component. The cross-coupling term $-L_{f} \vec{I}_{f} \frac{d \vec{E}_{i r p}}{d t}$ in IRP (shown in 
Eq.(22) and Eq.(24)) reveals that the general nature of the cross coupling term in SRF is a cross-differential phenomena, which can be explained as:

- When a time domain signal is shifted in the frequency domain, the shifted frequency will be reflected back to the time domain with one more cross - differential term.

- If the shifting is linear (one frequency or multiple frequency with the same size), the size of the cross differential term is proportional to the distance (or frequency), which is how much the signal is shifted in the frequency domain.

- If the shifting is nonlinear (multiple frequency with different size), the size of the cross - differential term is not proportional to the distance (or frequency), which is how much the signal is shifted in frequency domain, but is the result of the sum of shifted distance (or frequency) multipling their own size.

- In the non-linear situation, the portion of the different shifted frequency is not observable in time domain, so cross de-coupling in this case can not be realized for multiple frequency.

However, de-coupling control can still be realized for one frequency and this is the case when $E_{\text {irp }}$ only has one frequency component (fundamental).

\subsection{Direct Power Control}

Based on the connection between IRP and SRF, when $E_{\text {irp }}$ only has one frequency component(fundamental), Fig.4(d) and Fig.3 can be simplified into Fig.4(e) and Fig.5, which is proposed in this paper and can be called direct power control. Similar like Fig.4(d) and Fig.3, direct power control (Fig.5) generates a power reference and feedback (controlled) power based on the IRP theory. The error is fed into a power regulator and the output of the power regulator is then transformed back by the IRP to be the voltage reference of the inverter. The system is equivalent as a de-coupled synchronous/stationary hybrid current controller, but has a simpler structure. It also has a clear physical concept and can be generated by physical understanding of the system.
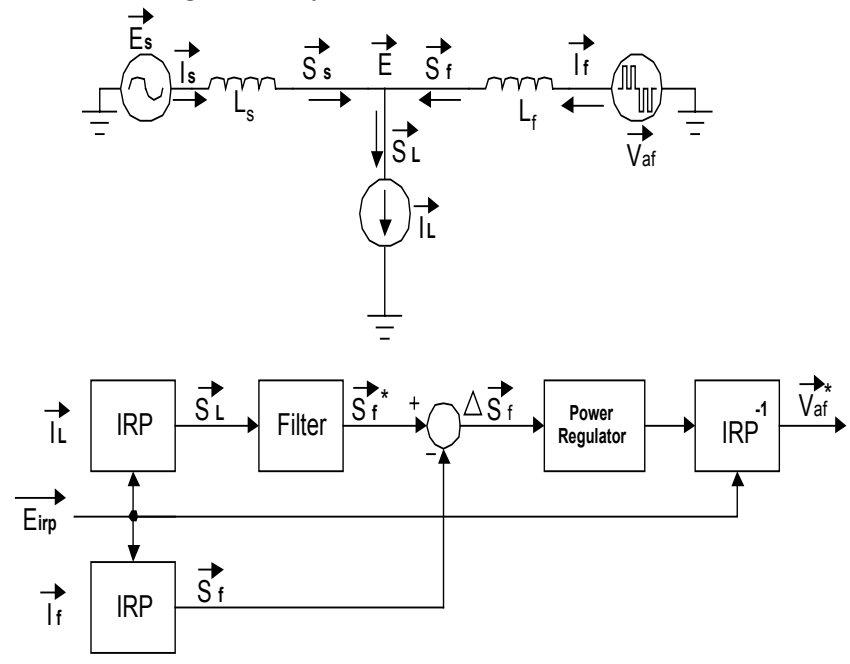

Fig.5 The proposed direct power controlled voltage source inverter based active power filter

\subsection{Simulations}

Simulations on a 4160V/1.2MVA active filter system are studied in Fig.6. Fig.6(a) demonstrates that the active filter current compensats the load current harmonics accurately in one cycle after starting up. Fig.6(b) shows that source current THD reduces from $25.7 \%$ to $4.6 \%$. Fig.6(c) shows the FFT of the load current and source current. It is obvious that the $5^{\text {th }}, 7^{\text {th }}, 11^{\text {th }}, 13^{\text {th }}$ four lower dominant harmonic are all eliminated as controlled. Fig.6(d) shows the switching pattern of the power devices which indicates that the power devices switch at $750 \mathrm{~Hz}$ as commanded.

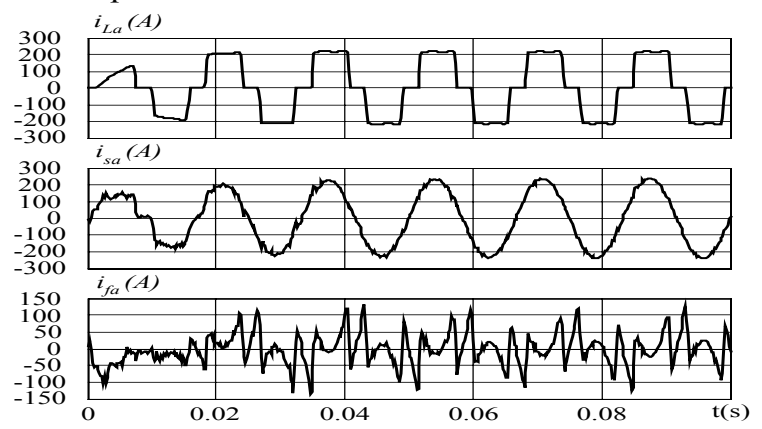

(a)Load current; source current; active filter current
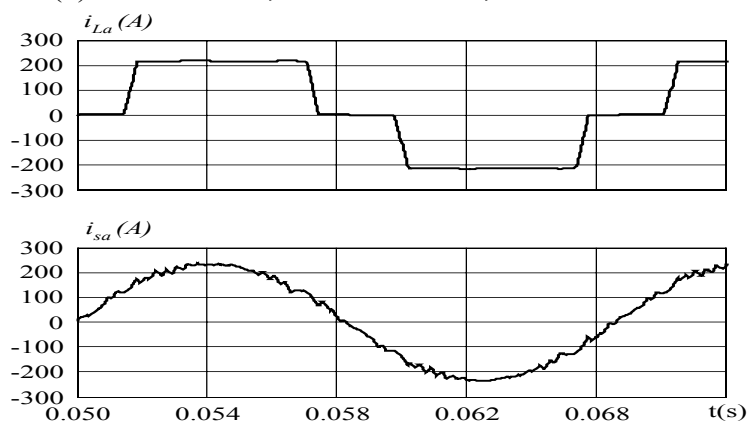

(b)Close waveforms of load current and source current

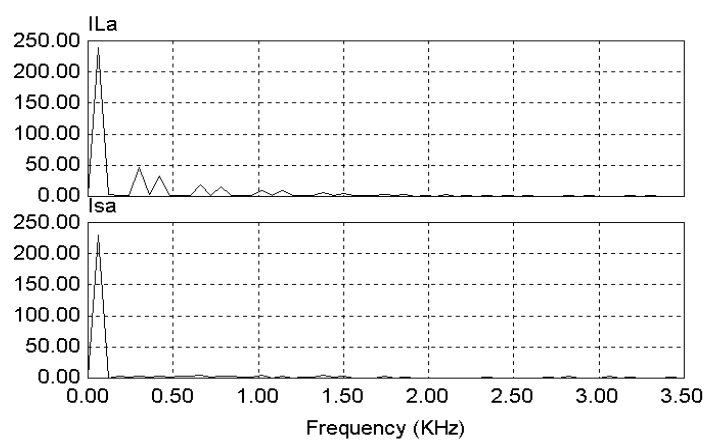

(c) FFT of load current ILa and source current Isa

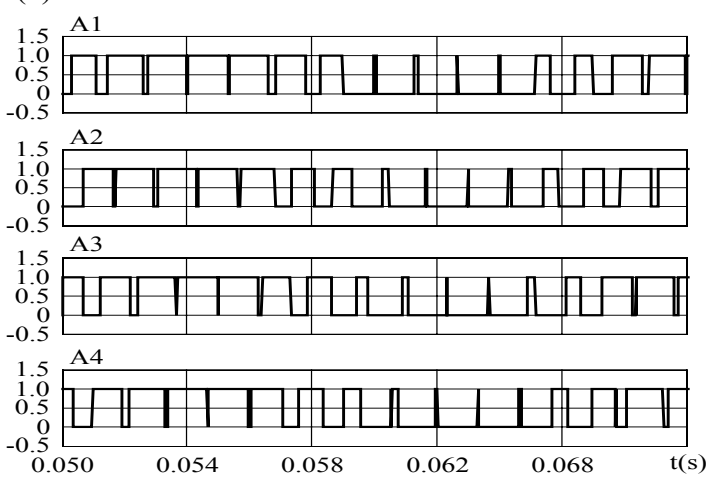

(d) One phase power device switching pattern 

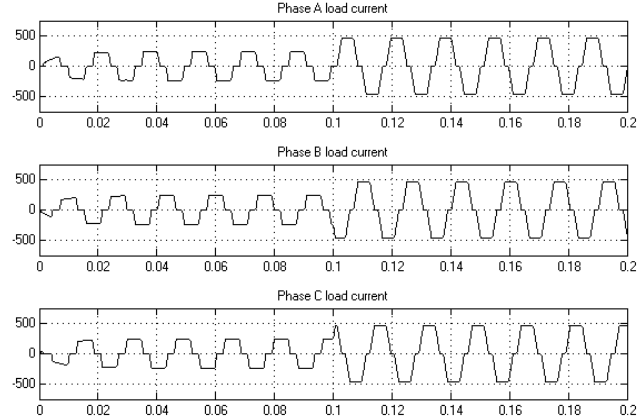

(e) Phase A,B,C load current

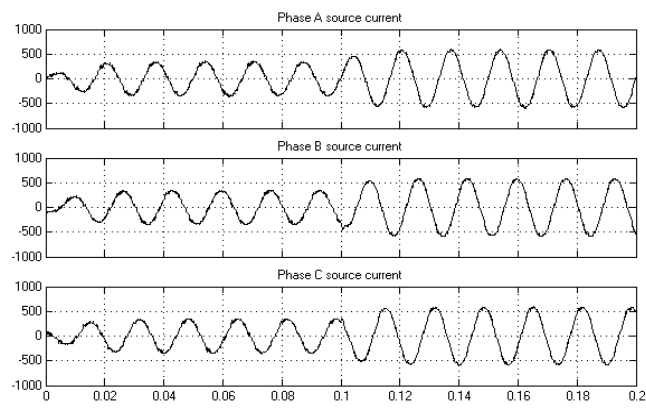

(f) Phase A,B,C source current

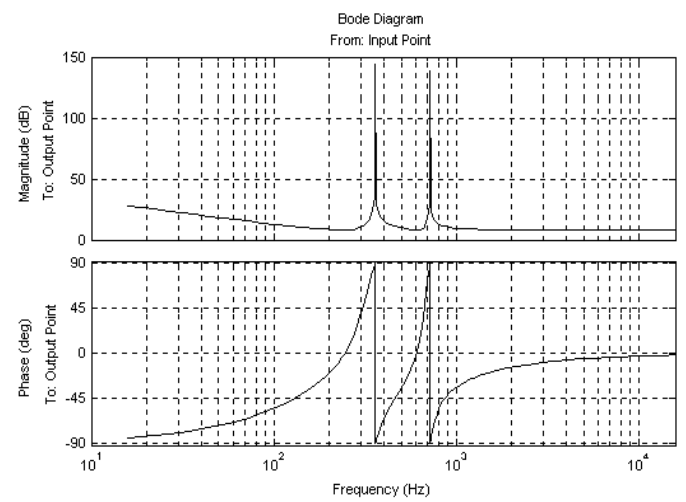

(g) Open loop bode plot of direct power controller

Fig. 6 Simulation results of the proposed multilevel inverter based direct power controlled active power filter

In Fig.6(e) and (f), DC load is changed in transient to produce a quasi-step change so that the dynamic response of the controller can be tested. With de-coupling control integrated in the proposed direct power controller, the load current can be compensated effectively in transients in Fig.6(f). For a diode rectifier balanced system as shown in Fig. 1 , only $-5^{\text {th }}, 7^{\text {th }},-11^{\text {th }}, 13^{\text {th }}$ harmonic exists and they are equivalents as $\pm 6^{\text {th }}$ and $\pm 12^{\text {th }}$ harmonic power when direct power control is applied. This can been seen in the open loop bode plot in Fig.6(g), where the equivalent feedforward open loop gain is going high at the frequency of compensated harmonics power with others almost unchanged. This means that the proposed direct power control can selectively increase the equivalent feedforward gain to reduce the error in the close loop for the compensated harmonic power, which is similar as the multiple frame current controller strucuture from which it is derived.

\subsection{Experiments}

A 6KVA lab prototype (Fig.7) was built with the same per unit value as shown in Fig. 1 by reducing voltage base value 20 times / current base value 10 times. The controller is based on a dSPACE DS1103 rapid prototyping system. The sampling rate is set at $12.5 \mathrm{KHz}$, and the switching frequency of the device is set as $625 \mathrm{~Hz}$ to reach an equivalent inverter switching frequency at $2.5 \mathrm{KHz}$. The experiment result is shown in Fig.8, which validates the proposed direct power control on the multilevel inverter based active power filter as the following:

- In Fig.8(a) and (c), after stating the active power filter, the source current is compensated effectively with power devices only switching at $625 \mathrm{~Hz}$.

- In Fig.8(b) and (d), the FFTs of the source current before and after starting active filter are shown. It is obvious that the $5^{\text {th }} 7^{\text {th }} 11^{\text {th }} 13^{\text {th }}$ harmonics are all eliminated and higher harmonics are almost unchanged. The THD of the source current drops from $28.2 \%$ to $4.8 \%$.

- In Fig.8(e) and (f), the system step response is tested with step-change of the load. It is shown that the decoupled control integrated within the proposed direct power control can effectively improve the dynamic performance of the system.

Based on the test results, the following can be concluded:

- With an equivalent $2.5 \mathrm{KHz}$ switching frequency (which is $625 \mathrm{~Hz}$ for power device at five-level design), $5^{\text {th }}, 7^{\text {th }}, 11^{\text {th }}, 13^{\text {th }}$ harmonics can be filtered effectively by the proposed active fiter concept.

- Very low ratios between switching frequency $(2.5 \mathrm{KHz})$ and controlled signal frequency (highest at $13^{\text {th }} 780 \mathrm{~Hz}$ ) is obtained (below 4), which greatly reduced the switching requirement and losses for the power device. The dynamic performance is also improved by the proposed direct power control at low switching frequency.

- With power device switching frequency around $625 \mathrm{~Hz}$, the proposed active filter is feasible in the medium voltage application, since the available power device IGCT in medium voltage range can only switch around $500-700 \mathrm{~Hz}$.

Overall, the proposed five-level active filter system is very attractive for medium voltage applications and this concept can be easily extended to higher level systems.

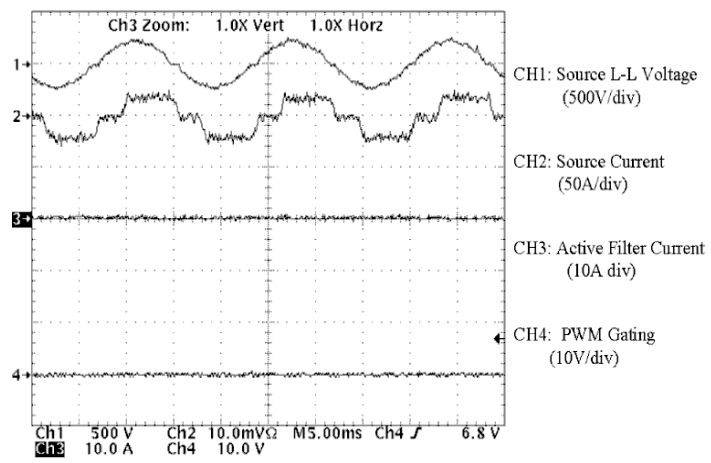

(a) Waveforms before the active filter is started 


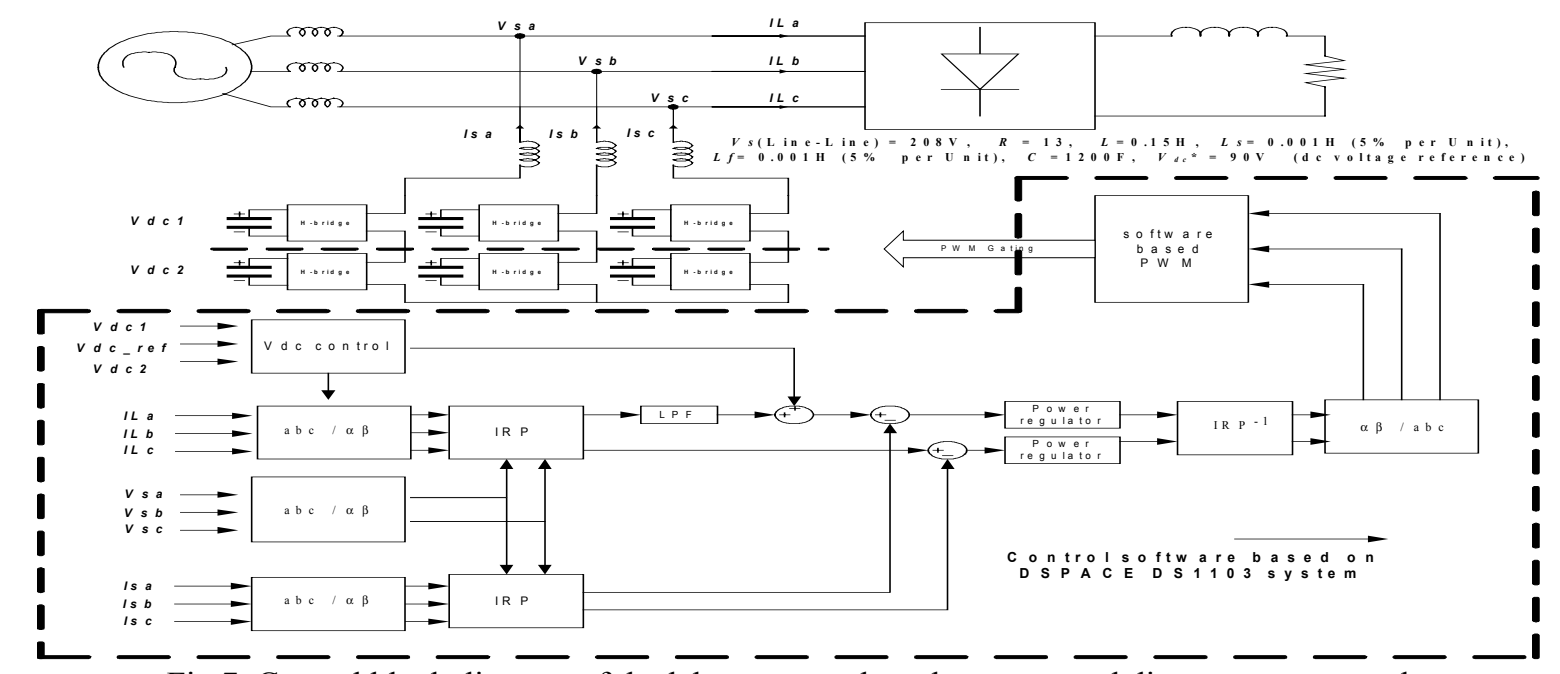

Fig.7 Control block diagram of the lab prototype based on proposed direct power control

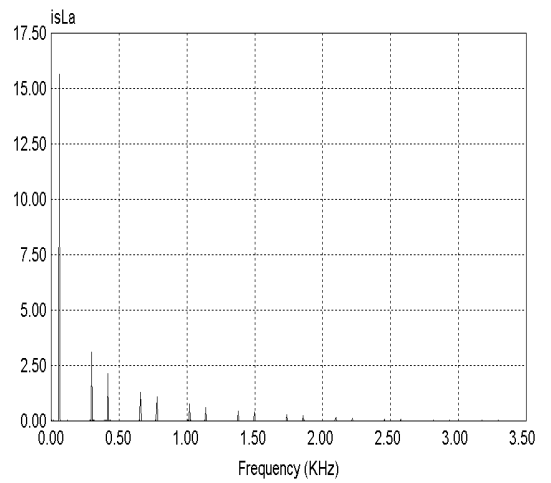

(b) FFT of source current before the actvie filter is started

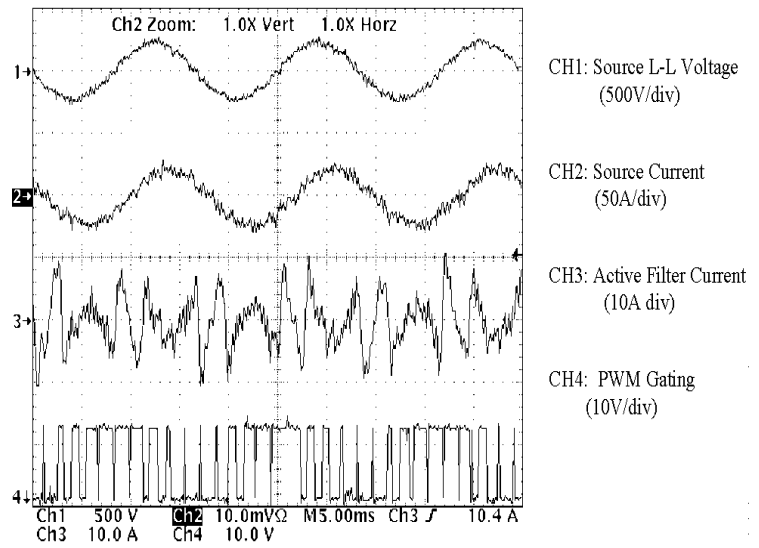

(c) Waveforms after the active filter is started

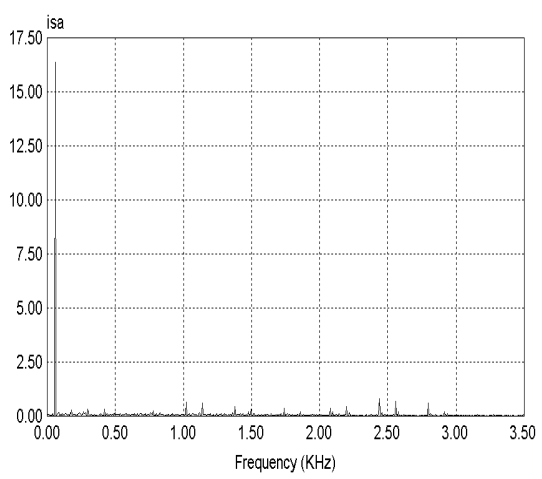

(d) FFT of source current after the actvie filter is started

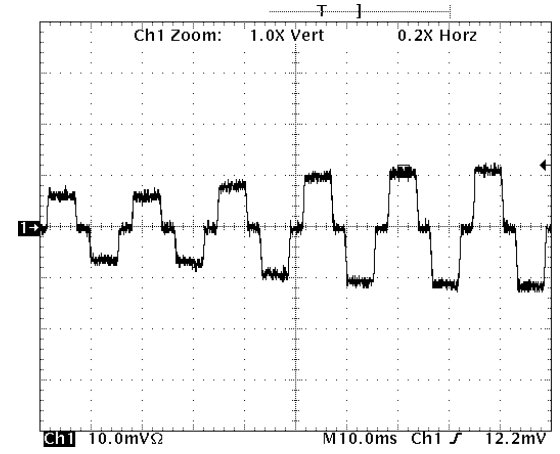

(e) Load current during system transient response

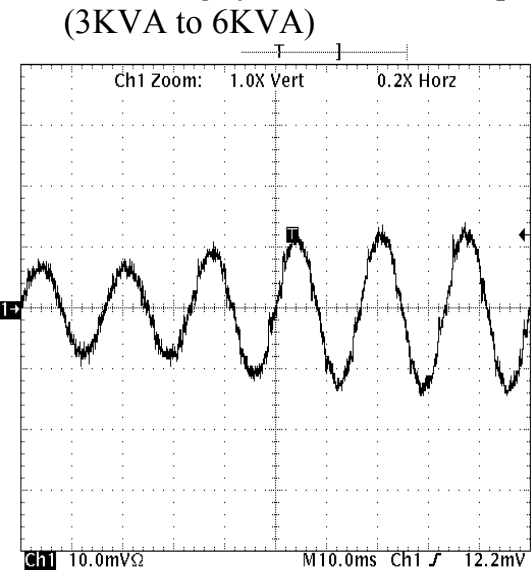

(f) Source current during system transient response ( $3 \mathrm{KV}$ to $6 \mathrm{KVA})$

Fig.8 6KVA lab prototype experimental results based on the proposed direct power control

\section{Conclusions}

A multilevel inverter based direct power controlled active power filter for medium voltage application is proposed in this paper. Derived from the multiple reference frame current controller, based on rotating frame and stationary frame, the proposed direct power control has a simplified structure and a clear physical meaning, which is equivalent as a de-coupled synchronous/stationary hybrid frame current controller. With a very low switching frequency, 
the proposed active power filter can effectively comepensate the dominant $5^{\text {th }}, 7^{\text {th }}, 11^{\text {th }}, 13^{\text {th }}$ harmonics from nonlinear load in steady and transient state, which makes it suitable for medium voltage application with improved bandwidth. This concept reveals some theoretical connections between IRP and SRF theories and can be considered as a bridge between them. Simulations on a $4160 \mathrm{~V} / 1.2 \mathrm{MVA}$ system and experimental results of a $208 \mathrm{~V} / 6 \mathrm{KVA}$ lab prototype are provided to prove the proposed theory.

\section{Appendix}

I. Rotation Frame to Stationary Frame Without Decoupling Control:

$$
\begin{aligned}
\vec{Y}(t) & =L^{-1}\left\{L\left[\vec{X}(t) * e^{-j \omega t}\right] * H_{\omega}(s)\right\} * e^{j \omega t} \\
& =L^{-1}\left[\vec{X}(s+j \omega t) * H_{\omega}(s)\right] * e^{j \omega t} \\
& =L^{-1}\left[\vec{X}(s+j \omega t-j \omega t) * H_{\omega}(s-j \omega t)\right] \\
& =L^{-1}\left[\vec{X}(s) * H_{\omega}(s-j \omega t)\right] \\
\Rightarrow & \vec{Y}(s)=\vec{X}(s) * H_{\omega}(s-j \omega t)
\end{aligned}
$$

If consider $\pm \omega$ rotating frame transformation

$$
\vec{Y}(s)=\vec{X}(s) *\left[H_{\omega}(s-j \omega t)+H_{-\omega}(s+j \omega t)\right]
$$

when $H_{\omega}(s)=H_{-\omega}(s)=\frac{1}{s}$

we will have

$$
\begin{aligned}
\vec{Y}(s) & =\vec{X}(s) *\left[\frac{1}{s-j \omega t}+\frac{1}{s+j \omega t}\right] \\
& =\vec{X}(s) *\left[\frac{s+j \omega t}{s^{2}+\omega^{2}}+\frac{s-j \omega t}{s^{2}+\omega^{2}}\right] \\
& =\vec{X}(s) * \frac{2 s}{s^{2}+\omega^{2}}
\end{aligned}
$$

II. Synchronous Reference Frame Based Control:

$$
\begin{aligned}
& \vec{V}=V_{\alpha}+j V_{\beta} ; \quad \vec{I}_{f}=I_{f \alpha}+j I_{f \beta} ; \quad \vec{E}=E_{\alpha}+j E_{\beta} \\
& \vec{V}-\vec{E}=L_{f} \frac{d \vec{I}_{f}}{d t} \\
& \vec{V} * e^{-j \omega t}-\vec{E} * e^{-j \omega t}=L_{f} \frac{d \vec{I}_{f}}{d t} * e^{-j \omega t} \\
& L_{f} \frac{d\left(\vec{I}_{f} * e^{-j \omega t)}\right.}{d t}=L_{f} \frac{d \vec{I}_{f}}{d t} * e^{-j \omega t}+L_{f} \vec{I}_{f} \frac{d e^{-j \omega t}}{d t} \\
& =L_{f} \frac{d \vec{I}_{f}}{d t} * e^{-j \omega t}+L_{f} \vec{I}_{f} *\left(-j \omega e^{-j \omega t}\right) \\
& =L_{f} \frac{d \vec{I}_{f}}{d t} * e^{-j \omega t}-j \omega L_{f} \vec{I}_{f} * e^{-j \omega t} \\
& =>L_{f} \frac{d \vec{I}_{f}}{d t} * e^{-j \omega t}=L_{f} \frac{d\left(\vec{I}_{f} * e^{-j \omega t)}\right.}{d t}+j \omega L_{f} \vec{I}_{f} * e^{-j \omega t} \\
& \vec{V} * e^{-j \omega t}-\vec{E} * e^{-j \omega t}=L_{f} \frac{d\left(\vec{I}_{f} * e^{-j \omega t}\right)}{d t}+j \omega L_{f}\left(\vec{I}_{f} * e^{-j \omega t}\right) \\
& \vec{V}_{\omega}=\vec{V} * e^{-j \omega t} ; \quad \vec{E}_{\omega}=\vec{E} * e^{-j \omega t} ; \quad \vec{I}_{f \omega}=\vec{I}_{f} * e^{-j \omega t} \\
& \vec{V}_{\omega}-\vec{E}_{\omega}=L_{f} \frac{d \vec{I}_{f \omega}}{d t}+j \omega L_{f} \vec{I}_{f \omega}
\end{aligned}
$$

III : Instantaneous Reactive Power Theory:$$
\vec{V}=V_{\alpha}+j V_{\beta} ; \quad \vec{I}_{f}=I_{f \alpha}+j I_{f \beta} ; \quad \vec{E}=E_{\alpha}+j E_{\beta}
$$

$\vec{E}_{i r p}=E_{\alpha}-j E_{\beta}$

$\vec{V}-\vec{E}=L_{f} \frac{d \vec{I}_{f}}{d t}$

$\vec{V} * \vec{E}_{i r p}-\vec{E} * \vec{E}_{i r p}=L_{f} \frac{d \vec{I}_{f}}{d t} * \vec{E}_{i r p}$

$L_{f} \frac{d\left(\vec{I}_{f} * \vec{E}_{i r p}\right)}{d t}=L_{f} \frac{d \vec{I}_{f}}{d t} * \vec{E}_{i r p}+L_{f} \vec{I}_{f} \frac{d \vec{E}_{i r p}}{d t}$

$=>L_{f} \frac{d \vec{I}_{f}}{d t} * \vec{E}_{i r p}=L_{f} \frac{d\left(\vec{I}_{f} * \vec{E}_{i r p}\right)}{d t}-L_{f} \vec{I}_{f} \frac{d \vec{E}_{i r p}}{d t}$

$\vec{V} * \vec{E}_{i r p}-\vec{E} * \vec{E}_{i r p}=L_{f} \frac{d\left(\vec{I}_{f} * \vec{E}_{i r p}\right)}{d t}-L_{f} \vec{I}_{f} \frac{d \vec{E}_{i r p}}{d t}$

$\vec{V}_{i r p}^{2}=\vec{V} * \vec{E}_{i r p} ; \vec{E}^{2}{ }_{i r p}=\vec{E} * \vec{E}_{i r p} ; \quad \vec{S}_{f}=\vec{I}_{f} * \vec{E}_{i r p}$

$\vec{V}_{i r p}^{2}-\vec{E}_{i r p}^{2}=L_{f} \frac{d \vec{S}_{f}}{d t}-L_{f} \vec{I}_{f} \frac{d \vec{E}_{i r p}}{d t}$

when $\vec{E}_{i r p}=\sqrt{E_{\alpha}^{2}+E_{\beta}^{2}} e^{-j \omega t}$ IRP based equation will become SRF based equation.

$$
\begin{aligned}
& \text { If } \vec{E}_{i r p}=\sum_{k=1}^{n} E_{k} e^{-j k \omega t} \\
& =>\quad-L_{f} \vec{I}_{f} \frac{d \vec{E}_{i r p}}{d t}=L_{f} \sum_{k=1}^{n}\left[\left(j k \omega E_{k}\right) *\left(\vec{I}_{f} e^{-j \omega t}\right)\right]
\end{aligned}
$$

\section{REFERENCES}

[1] Jin-Sheng Lai, Fang Zheng Peng, "Multilevel Converters - a New Breed of Power Converters", IEEE Trans. Industry Applications, Vol.32, No.3 pp509-517, 1996.

[2] Walker G.,Ledwich, G., "Bandwidth Considerations For Multilevel Converters", IEEE Trans. Power Electronics, Vol.14, No.1, pp74-81, 1999.

[3] Daniel Nahum Zmood, Donald Grahame Holmes, Gerwich H. Bode "Frequency-Domain Analysis of ThreePhase Linear Current Regulators"IEEE Trans. On Industry Application, vol.37, no.2, March/April 2001

[4] Xiaoming Yuan, Willi Merk, Herbert Stemmler, and Jost Allmeling "Stationary-Frame Generalized Integrators for Current Control of Active Power Filters With Zero Steady-State Error for Current Harmonics of Concern Under Unbalanced and Distorted Operating Conditions" IEEE Trans. On Industry Application, vol.38, no.2, March/April 2002

[5] Hirofumi Akagi, "New Trends in Active Filters for Power Conditioning", IEEE Trans. Industry Applications, Vol.32, No.6, pp1312-1322, 1996.

[6] Simone Buso, Luigi M., Paolo M., "Comparison of Current Control Techniques for Acive Filter Applications", IEEE Trans. Industry Elecronics, Vol.45, No.5 pp722-729, 1998.

[7] Jost Allmeling, "A Control Structure for Fast Harmonics Compensation in Active Filters" IEEE Trans. On Power Electronics, vol.19, no.2, March 2004 